Western University

Scholarship@Western

Mechanical and Materials Engineering

Mechanical and Materials Engineering

Publications

Department

2015

\title{
A new approach to digital generation of spherical void phase porous media microstructures
}

Nolan J. Dyck

The University of Western Ontario

Anthony G. Straatman

uwo, agstraat@uwo.ca

Follow this and additional works at: https://ir.lib.uwo.ca/mechanicalpub

Part of the Materials Science and Engineering Commons, and the Mechanical Engineering Commons

Citation of this paper:

Dyck, Nolan J. and Straatman, Anthony G., "A new approach to digital generation of spherical void phase porous media microstructures" (2015). Mechanical and Materials Engineering Publications. 18.

https://ir.lib.uwo.ca/mechanicalpub/18 


\title{
A new approach to digital generation of spherical void phase porous media microstructures
}

\author{
Nolan J. Dyck, Anthony G. Straatman* \\ Department of Materials and Mechanical Engineering, The University of Western Ontario, 1151 Richmond St. London, Ontario, Canada
}

\section{A R T I C L E I N F O}

\section{Article history:}

Received 24 April 2014

Received in revised form 7 October 2014

Accepted 9 October 2014

\section{Keywords:}

Digital generation

Bulk property prediction

Porous media

Carbon foam

\begin{abstract}
A B S T R A C T
This work describes a novel approach for obtaining digital samples of porous media based on a statistical knowledge of the microstructure of interest. The present formulation introduces a contact law based on bubble physics that is capable of handling interferences among spherical primitives of different diameter placed in a representative elemental volume, while the volume is compressed to yield a target porosity. The result is a statistically accurate mathematical model of a permeable, spherical-void-phase porous material that has the added feature of being spatially periodic in all principal directions. To validate the approach, digital samples of spherical-void-phase carbon foam were generated and discretized for use in hydraulic and thermal Computational Fluid Dynamics simulations. Relevant transport properties were computed from the simulation results, and compared to similar data found in the literature.
\end{abstract}

(c) 2014 Elsevier Ltd. All rights reserved.

\section{Introduction}

Porous materials play an important role in many applications including heat pipes, heat sinks, automotive cooling devices and solar collectors. Any porous microstructure may be characterized by the statistical geometric properties: mean pore diameter, ligament length, surface area per unit volume, void shape, and geometric order, each of which might be correlated with changes in bulk material properties. The microstructural properties also affect exchanges that occur between fluid and solid phases in the case of flow through a permeable porous material, or in the case of external exposure to incident radiation. This has inspired many researchers to study the effects of pore structure variation on bulk material properties by developing representative, or idealized, geometric models. Such models permit analysis of properties and exchanges at the pore level, which can then be used to develop accurate mathematical models at the porous-continuum or volume-averaged level, which is what is used in most engineering analyses to deal with porous materials. In this paper, our interest is to develop a geometric model for spherical-void-phase (SVP) carbon foam that can be used to study exchanges that occur in a highly concentrated solar collector. Our interest in graphitic foam is that it is highly permeable, highly conductive and it has a high surface area to volume ratio. While pore-level geometric models do exist for spherical void phase materials, no current model is

\footnotetext{
* Corresponding author. Tel.: +1 (519) $6612111 \times 88249$.

E-mail address: astraatman@uwo.ca (A.G. Straatman).
}

suitable to yield the material properties and exchanges, while preserving the random nature of the structure, which is required for consideration of radiation effects.

Currently, there are three main approaches to obtain bulk material properties, given the porous material of interest. First, experiments can be conducted to empirically determine the desired properties. While this method is quite useful for determining properties that can be used in engineering calculations, it can be difficult for researchers to understand the influence of the microstructure on the determined properties. Moreover, because experiments can only be performed on physical samples, the range of microstructures that can be studied is limited by current production capabilities.

The second method is to conduct Computer Tomography (CT) scans of a representative sample of the microstructure, thereby obtaining a digital representation of the structure. The raw data from the scan is post-processed to obtain a Computer-Aided Design (CAD) model. This model can then be used in computer simulations to determine the desired transport properties. Researchers including Haussener et al. [1], Maruyama et al. [2], and Anghelescu [3] have used this approach with some success. Unlike the experimental approach, this method allows researchers to visualize transport quantities and gradients throughout the pore level domain. However, the cost and accessibility of CT scanning equipment as well as the limitation of porous media production capabilities leaves more to be desired.

The final method is to generate a large number of small primitive objects (primitives) within a finite domain, where the 
dimensions of the primitives are decided based on statistical data describing the microstructure of interest. The interference of these primitives gives rise to the interesting features present in the porous domain. A finite volume filled with interfering primitives is thought to be an accurate representation of the desired microstructure. These digital domains can then be used in small-scale simulations to predict effective material properties. This method is unique in the fact that it does not require any physical representation of the domain, but only a statistical knowledge of the micro-structural features. Because this method deals with idealized representations of porous media, it is important to judge the relevance of all microstructural features to the bulk property of interest. Identifying which microstructural features whose variation does not influence a bulk property may allow a much simpler geometric model to be used. A first attempt by Yu et al. [4] constitutes the use of a simple primitive: the Boolean subtraction of a sphere from a cube (where the centroids of the sphere and cube are coincident). This primitive is stacked along all major axis to obtain a uniform structure of interconnected pores. This so-called unit-cube model is easy to generate and analyze digitally, however it does not capture the effect of bubble size variation, and it exhibits uncharacteristic properties due to the axis alignment of the bubbles. When used for studying trends in convective/conductive heat transfer and fluid flow, the unit cube model is sufficient, as predictions of permeability, inertial drag coefficients, and Nusselt number correlations were shown to be reasonable when the flow direction was oriented $45^{\circ}$ from all principle planes [4,5]. Kumar et al. [6] performed Computational Fluid Dynamics (CFD) analyses involving the periodic tetrakaidecahedron structure (Kelvin cell) relevant to open-cell foams. The CFD results were compared with experimental results obtained using a uniform microstructure, which yielded excellent agreement. Leong and Li [7] determined the effective thermal conductivity of a unit cell which may be described as the Boolean subtraction of eight spheres from a cubic shell, where the spheres are centered on the vertices of the cube. James et al. [8] presented a novel approach for the generation of spherical-void phase (SVP) porous media wherein bubble diameters and interferences are chosen based on known probability distribution functions. That said, the method presented in [8] has some drawbacks, including the unrealistic assumption that the interference between two bubbles in contact is independent of their radii, and the random deletion of bubbles to obtain the desired porosity. In another work by Kirca et al. [9], carbon foam was modeled by selecting random points within a cubic domain as the sphere centers, and calculating the radii of the bubbles based on the desired porosity and the average bubble diameter. The method also suffered from the fact that bubble interferences were not determined. Wang and Pan [10] have proposed a random-generation growth method, wherein representations of open-cell foams are generated by randomly placing points in a 3D domain and stochastically linking neighboring nodes. Finally, in a recent publication by Chueh et al. [11], SVP was modeled by packing spheres into a periodic domain using the drop-and-roll method introduced by Visscher and Bolsterli [12]. This method relies on the assumption that bubble interferences can be prescribed using a constant contact angle, and periodicity is only enforced in two directions.

The primary motivation for developing the present method is to obtain digital samples of SVP carbon foam suitable for prediction of radiation transport properties. The ordered nature of the unit-cube model makes it a poor candidate for ray-tracing methods, such as that presented by Tancrez and Taine [13], so more accurate representations of SVP porous media are sought. In this work, the approach taken is similar to previous efforts, however in the Representative Elemental Volumes (REVs) produced using the current method, the interferences between features are governed by physically-based force-displacement relationship, and the REVs have the distinct advantage of being fully spatially periodic. The resulting models are then shown to produce the correct trends for pressure drop and convective heat transfer, thereby satisfying the necessary condition that fluid-solid interactions within the REV are properly predicted. Combined with the advantages of including variable pore size and preserving the random nature of the structure, the present modeling approach is considered most viable for studies on incident radiation.

\section{Formulation}

In the current approach, a 3D porous structure is generated from aggregate statistical data. For example, an isotropic SVP porous medium may have a statistical data set including an average bubble diameter and the standard deviation of the bubble diameters.

Once these data are determined, the method can be executed with the following steps:

(1) Select an Initial Volume (IV) shape and size. The IV should be large enough to completely envelop all of the geometric primitives without any of the primitives coming into contact with each other.

(2) Generate primitives. Choose the shape and dimensions of each primitive using a probabilistic model that reflects the statistical data. Then attempt to randomly locate each primitive within the IV, optionally choosing an orientation based on statistical data. If a primitive interferes with an already placed primitive, randomly select new locations and orientations until it does not interfere with other primitives. If a primitive cannot be placed after a large number of attempts, this may indicate that the IV is too small, in which case the algorithm should be restarted with a larger IV. The stopping criterion is at the discretion of the user. A simple approach is to stop after a pre-determined number of primitives have been placed.

(3) Using a Discrete Element Method (DEM) code, incrementally compress the IV over a sequence of time-steps. During each time-step, the domain boundaries are moved closer together. If the boundaries are chosen to be rigid walls, the primitives in contact with the boundaries will be forced inward, and come into contact with other primitives. Reaction forces (or separating forces) between primitives in contact are calculated using an appropriate force-displacement relationship, also known as a contact law. Eventually the primitives will reach a "jammed" state, where every primitive is locked in place. After the jammed state has been reached, further compression steps require ever-increasing forces to be applied to the walls, while the penetrations between primitives in contact are also increased. The stopping criterion is at the discretion of the user. A simple criterion would be to stop when the desired porosity is reached.

While rigid walls may be easy to implement, the resulting domains will not be periodic; a desirable trait for subsequent prediction of volume averaged properties. To obtain periodic domains, the rigid walls must be replaced with periodic boundary conditions. Periodic boundary conditions can be implemented by 'wrapping' the coordinates of all the primitives inside the domain, a concept illustrated in Fig. 1.

In Fig. 1, Primitive $A$ is wrapped across a flat periodic interface in the $x$-direction to form primitive $A^{\prime} . A^{\prime}$ acts as a proxy for $A$; the collision between $A^{\prime}$ and $B$ is treated as a collision between $A$ and $B$. In contrast to a rigid wall the periodic boundary does not apply a direct force to the primitives in contact. If the domain is 


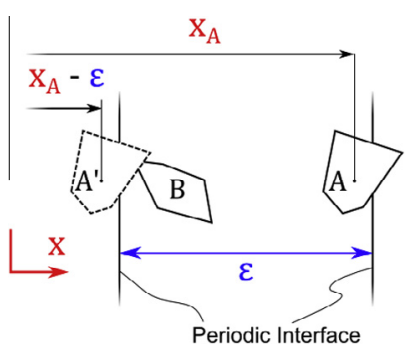

Fig. 1. Periodic interface implementation showing two original primitives, $A$ and $B$ as well as one wrapped primitive, $A^{\prime}$. The distance between the two periodic faces is $\varepsilon$.

compressed by some distance $\Delta \varepsilon$, the co-ordinate of $A^{\prime}$ will increase by the same amount. This in turn will increase the penetration of the contact between $A^{\prime}$ and $B$. As a consequence, the separation force between $A$ and $B$ will also increase.

(4) Extract the dimensions, locations, and (optionally) orientations of the primitives, and use the data to generate the CAD model.

\section{Contact law}

Because the current method makes use of the DEM, the interaction between primitives (e.g. particles, fibers, bubbles) in contact is dependent on a known contact law. A contact law may be defined based on the materials of the two primitives in contact as well as their geometries. In preparation for the generation of SVP porous media samples, a contact law governing the interaction of bubbles will be derived.

Carbon foam may be manufactured by bubbling molten tar pitch [14]; during the packing phase initially sparse bubbles expand, and are forced into contact with one another as the domain is saturated. When the domain is packed, the pitch is cooled and the bubbles are frozen in place. It is of interest to model the packing phase in a DEM simulation, so a contact law governing the interaction of bubbles will be derived.

The setup in Fig. 2 has been analyzed by Chan et al. [15] who derived the general force-displacement relationship:

$$
\begin{aligned}
\Delta D \cong & \frac{F}{4 \pi \sigma_{1}}\left\{\ln \left(\frac{F \bar{R}_{o}}{8 \pi \bar{\sigma} R_{o 1}^{2}}\right)+2 B\left(\theta_{o 1}\right)\right\} \\
& +\frac{F}{4 \pi \sigma_{2}}\left\{\ln \left(\frac{F \bar{R}_{o}}{8 \pi \bar{\sigma} R_{o 2}^{2}}\right)+2 B\left(\theta_{o 2}\right)\right\}-\frac{F}{2 \pi \bar{\sigma}}
\end{aligned}
$$

$\frac{1}{\bar{R}_{o}} \equiv \frac{1}{2}\left(\frac{1}{R_{o 1}}+\frac{1}{R_{o 2}}\right)$

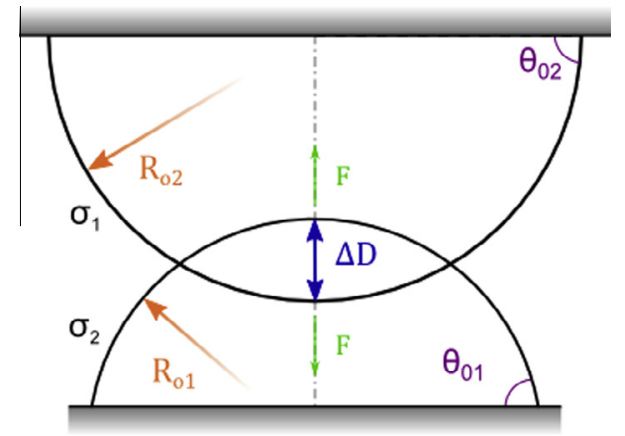

Fig. 2. Axis-symmetric opposing bubbles affixed to parallel surfaces. The overlapping region is called the interaction zone.
Table 1

Descriptions of terms in Eq. (1).

\begin{tabular}{ll}
\hline$\Delta D$ & Interference (penetration) between interacting bubbles \\
\hline$F$ & Normal force acting along the line of contact between the two \\
& bubbles \\
$\sigma_{1}, \sigma_{2}$ & Surface tension of the fluid surrounding each bubble \\
$R_{o 1}, R_{o 2}$ & Undeformed radius of each bubble \\
$\theta_{o 1}, \theta_{o 2}$ & Contact angle of each undeformed bubble \\
\hline
\end{tabular}

$\frac{1}{\bar{\sigma}}=\frac{1}{2}\left(\frac{1}{\sigma_{1}}+\frac{1}{\sigma_{2}}\right)$

where the parameters in Eqs. (1)-(3) are defined in Table 1.

Two separate forms of the function $B(\theta)$ were proposed: in the first case the contact angle $\theta$ was assumed to remain constant as the bubbles are depressed. In the second case, the contact line of each bubble with the wall is assumed fixed, while $\theta$ is allowed to vary during the deformation. Only the first case is relevant to the present application, where $B(\theta)$ has the form:

$B(\theta)=1+\frac{1}{2} \ln \left(\frac{1+\cos \theta}{1-\cos \theta}\right)-\left(\frac{1}{2+\cos \theta}\right)$

In order to apply Eq. (1) to bubbles in SVP porous media, a crucial observation must be made: deformation of a bubble having a constant wall contact angle of $90^{\circ}$ is identical to the case where a spherical bubble suspended in a liquid is opposed on either side by bubbles of equal radii. In other words, if a constant contact angle of $90^{\circ}$ is assumed for either bubble in Fig. 2, the solid wall behaves as a symmetry plane. Building on this realization, it can be concluded that Eq. (1) also applies to the bubbles in Fig. 3.

The bubbles in Fig. 3 bear some resemblance to interacting bubbles in SVP porous media; however, bubbles in porous media often come into contact with three or more bubbles with differing radii. In such cases the loading is no longer confined to a single axis, violating the axis-symmetry assumption made by Chan et al. [10]. In lieu of an arduous derivation of a more general force-displacement relationship, Eq. (1) is thought to be an adequate contact law for usage in a DEM framework, subject to assumptions: (a) interacting bubbles retain their spherical shape outside the interaction zone, (b) their initial radii remain unchanged during any interactions, and (c) all fully-immersed bubbles interact as if they were affixed to a wall with $\theta_{o}=90^{\circ}$. Inserting assumption (c) into Eq. (4) yields $B\left(\theta_{01}\right)=\frac{1}{2}$. Using this information, Eq. (1) can be simplified:

$\Delta D \cong \frac{F}{4 \pi}\left[\frac{1}{\sigma_{1}} \ln \left(\frac{F \bar{R}_{0}}{8 \pi \bar{\sigma} R_{o 1}^{2}}\right)+\frac{1}{\sigma_{2}} \ln \left(\frac{F \bar{R}_{0}}{8 \pi \bar{\sigma} R_{o 2}^{2}}\right)\right]$

Often, the molten pitch can be considered homogenous, and the surface tension acting around every bubble can be considered equal. Mathematically, this means $\sigma=\sigma_{1}=\sigma_{2}$. Substituting this expression into Eq. (5) results in:

$\Delta D \cong \frac{F}{2 \pi \sigma} \log \left(\frac{F}{8 \pi \sigma R_{A V G}}\right)$

$R_{A V G}=\frac{R_{o 1}+R_{o 2}}{2}$

As indicated by [16], Eq. (1) (and by extension Eq. (6)) is valid only when (a) viscous forces are small in comparison to surface tension forces and (b) the inequality

$\frac{F}{2 \sigma \pi R_{A V G}} \ll 1$

is satisfied. Inequality (8) arises from the assumption that the radius of the contact area is small compared to the radius of the bubble. The left hand side of inequality (8) will hereby be referred to as 


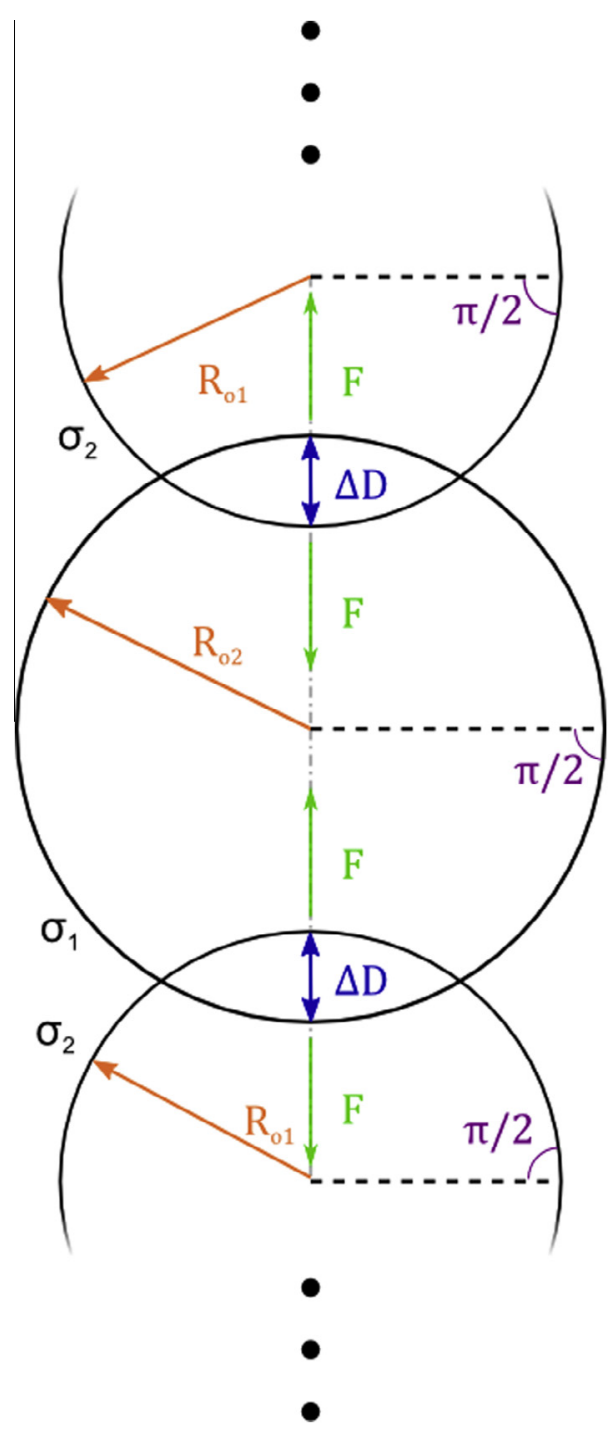

Fig. 3. An infinite linear chain of interacting spherical bubbles with alternating radii.

the force ratio. Constraint (a) is satisfied for SVP porous media as the relative motion of bubbles is small during the solidification process. Constraint (b) must be verified on a case-by-case basis.

\section{Validation}

To validate the method and the contact law from Section 3, 12 distinct carbon foam REVs were generated using the current method. Geometric statistical data provided by Oak Ridge National Laboratories [14] were used. A CFD analysis was then performed on one REV using ANSYS-CFX ${ }^{\mathrm{TM}}$ software [17], and the results were post-processed to obtain predictions of the permeability, Forchheimer coefficient, and the Nusselt number correlation of the porous material.

\subsection{Digital sample generation}

The steps to generate digital REVs were completed using an open-source DEM framework called Yet Another Development Engine (YADE) [18]. YADE is frequently used to study solid particle packing problems with deformable particles, making it well-suited for the present application.
In SVP porous media, the primitives are assumed to be spherical bubbles whose interaction is governed by the contact law, Eq. (6). YADE employs a penalty method when solving contacts (calculating a reaction force based on the contact interference at each time-step), so it is necessary to solve Eq. (6) for the contact force. To accomplish this, the Newton-Raphson method was used to evaluate the force iteratively. Convergence was achieved when the change in force between successive iterations was less than $0.1 \%$.

The number of bubbles in an REV, $N$, was varied from 100 to 400 by increments of 100 . Three REVs were generated for each value of $N$, so that 12 REVs were generated in total.

For each simulation, the steps outlined in Section 2 were followed:

(1) The IV was chosen to be a cube, occupying five times the total volume of $N$ bubbles: $V_{I V}=5 N V_{A V G}=5 N\left(\frac{4}{3} \pi R_{A V G}^{3}\right)$

(2) $N$ bubbles were generated. Diameters were selected from a normal distribution having a mean pore diameter of $400 \mu \mathrm{m}$ and a standard deviation of $120 \mu \mathrm{m}$ [14].

(3) YADE was used to compress the IV. Periodic boundary conditions were enforced throughout the simulation. A surface tension coefficient of $0.035 \mathrm{~N} / \mathrm{m}$ [19] was assumed for molten tar-pitch. During this step every contact was checked at regular intervals during compression to ensure $F /\left(2 \pi \sigma_{0} R_{A V G}\right)<0.1$. The simulation was halted when the porosity of the cube reached $80 \%$.

(4) Upon completion of the simulation, the sphere locations and sizes were exported to a text file. The text file was then read by a Visual Basic macro, which recreated the geometry in the 3D CAD software package, Solidworks ${ }^{\mathrm{TM}}[20]$. An image of the CAD geometry of one REV can be seen in Fig. 4 .

Fig. 4 depicts an REV produced using the current method. The model could be used in a structural simulation to estimate material properties such as elastic modulus or yield strength. If the geometry is subtracted from a cube of the same edge length, the resulting negative volume represents a fluid domain, and may be used to predict transport properties useful in CFD calculations, such as permeability, Forchheimer coefficient, and interfacial heat transfer coefficient.

As Fig. 5 shows, geometries produced using the current method bear a strong resemblance to their physical counterparts, capturing the random nature of the pores.

For each simulation, the average force ratio was evaluated upon completion and the results can be seen in Fig. 6. These values were considered to be small enough to satisfy inequality (8).

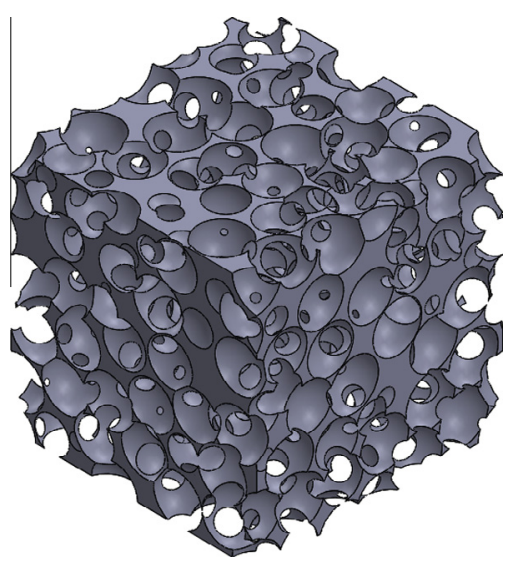

Fig. 4. CAD isometric view of a REV of graphite foam sample. 


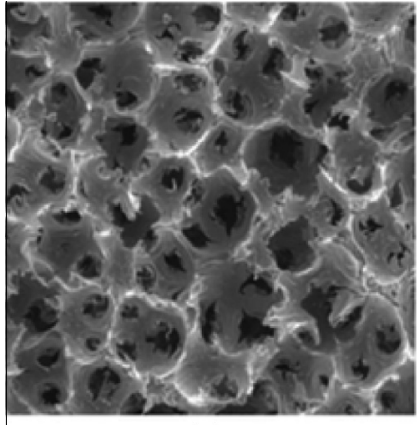

(a)

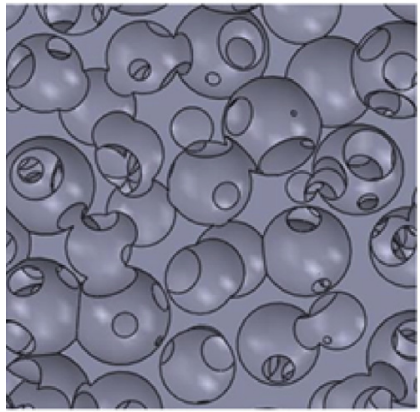

(c)

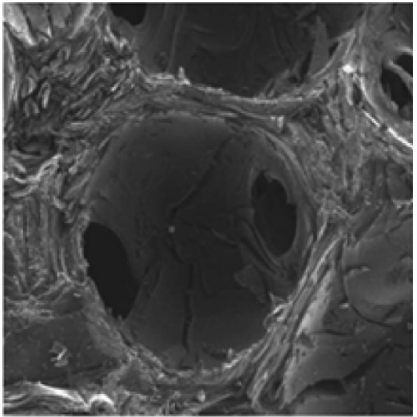

(b)

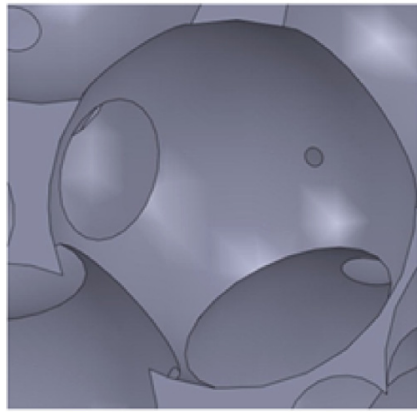

(d)
Fig. 5. Electron micrograph images of a graphite foam specimen (a) and (b) [21] in comparison to a CAD model of geometry generated using the current method (c) and (d).

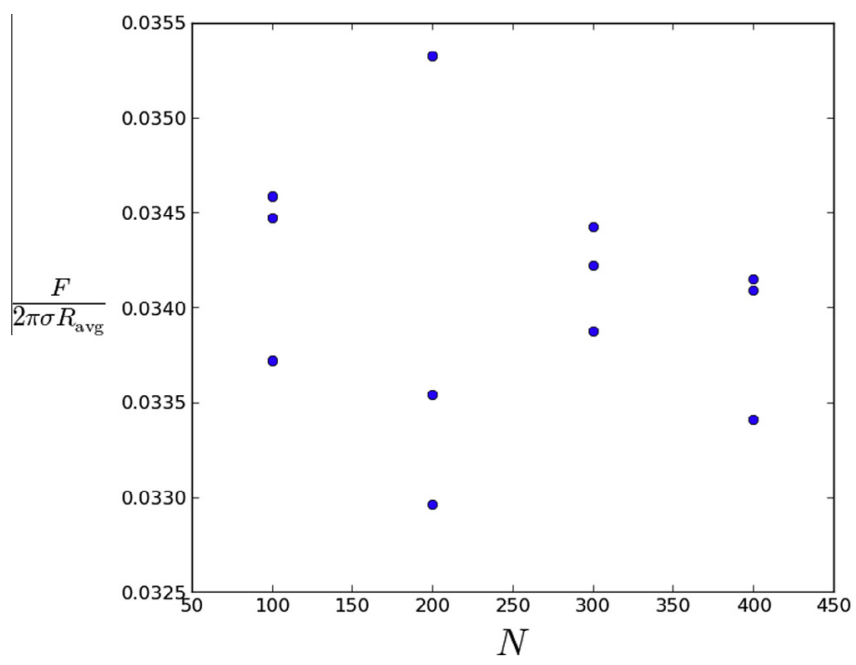

Fig. 6. Average force ratio vs. number of primitives.

\subsection{Setup}

Recall that the objective of this work was to generate porous media samples with the intent of extracting desired volume-averaged transport properties. While the DEM simulations produce geometries that visually resemble their physical counterparts, they have not yet been shown to have similar transport properties. To this end, a set of CFD simulations have been conducted using a 100 -sphere, $80 \%$ porosity REV from the previous section to determine the permeability, Forchheimer coefficient and convection heat transfer coefficients.

\subsubsection{Problem setup}

Using ANSYS ${ }^{\circledast 2}$ CFX $^{\mathrm{TM}}[17]$, inlet and opening boundary conditions were applied to opposite faces the REV, and a mass flow rate was imposed at the inlet boundary. Translationally periodic interfaces have been set up for the remaining two pairs of faces.

The hydraulic and energy equations were solved simultaneously; buoyant forces were neglected. A constant wall temperature of $393 \mathrm{~K}$ was prescribed, and the bulk inlet temperature was $293 \mathrm{~K}$. Energy increase due to viscous dissipation was also neglected.

Because the geometry was assumed statistically isotropic, three sets of simulations were run using the same REV (enforcing the desired mass flow rate across the $x, y$, and $z$ faces, consecutively) to obtain extra data for comparison.

\subsubsection{Computational setup}

To prepare the simulations, the geometry was first imported into ANSYS Workbench, where the model was meshed using the ANSYS $^{\text {}}$ Meshing ${ }^{\mathrm{TM}}[22]$ tool. Fig. 7 shows images of the resulting mesh composed of 2,932,590 nodes and 16,435,045 elements.

The working fluid is generic and was prescribed constant properties of $\rho=1000 \mathrm{~kg} / \mathrm{m}^{3}, \quad \mu=0.001 \mathrm{~N} \mathrm{~s} / \mathrm{m}^{2}, k=0.6 \mathrm{~W} / \mathrm{m} \mathrm{K}$, and $c_{p}=4184 \mathrm{~J} /(\mathrm{kg} \mathrm{K})$. The results were computed on a PC with an Intel i7 processor, utilizing 4 cores. Each simulation required approximately $30 \mathrm{~min}$ to converge the root mean square residuals within $1 \times 10^{-4}$.

\subsubsection{Grid independence}

To demonstrate grid independence, the domain was discretized with a finer grid density to yield $6,174,702$ nodes, and the $\operatorname{Re}=80$

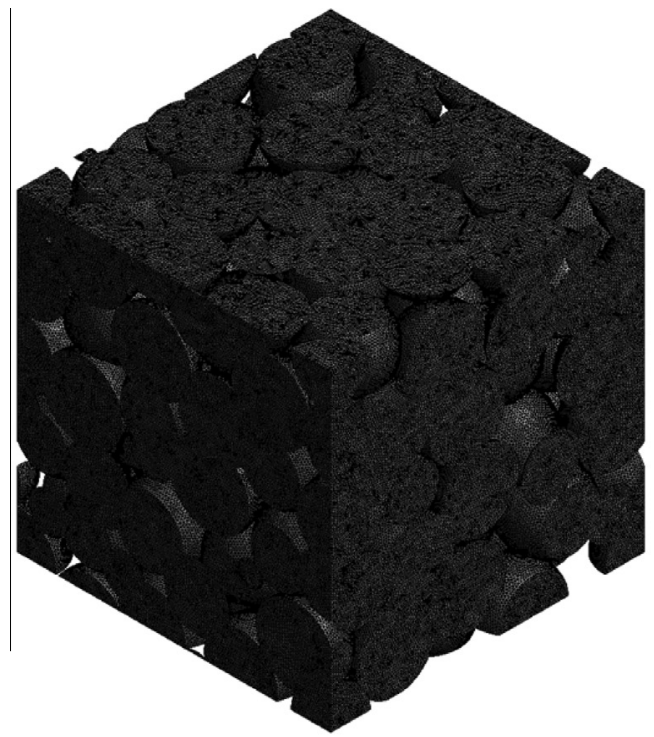

(a)

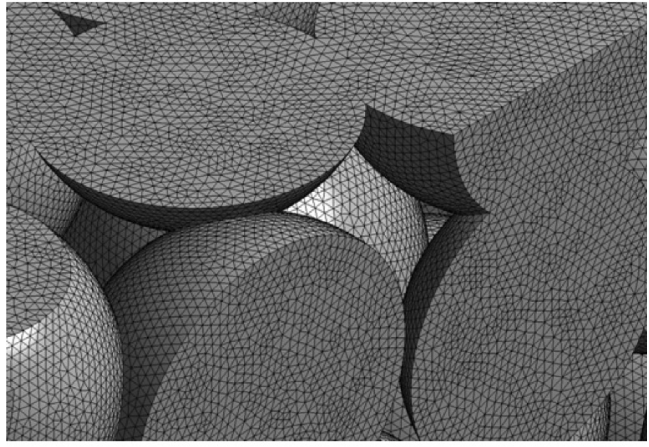

(b)

Fig. 7. ANSYS generated unstructured, tetrahedral mesh showing (a) broad-view and (b) close-up. 
case was re-run. The overall pressure drop was observed to be grid independent within $4.55 \%$, while the average wall heat flux was observed to be grid independent within 5.38\%.

\subsection{Results}

\subsubsection{Momentum results}

To observe the pressure drop within the Darcy regime and the stationary flow regime, the Reynolds numbers considered are $\operatorname{Re}_{d} \in\{0.1,0.5,1,10,40,60,80\}$.

The Darcy-Forchheimer Law may be stated as [23]:

$\frac{\Delta P}{L}=\frac{\mu}{K} U+\frac{c_{f}}{\sqrt{K}} \rho U^{2}$

where $\Delta P$ is the pressure drop, $L$ is the edge length of the REV, $U$ is the extrinsic velocity, $K$ is the permeability and $c_{f}$ is the Forchheimer coefficient. The polynomial fit function available in the python module, numpy.polyfit [24] was used to fit the results to Eq. (9). Fig. 8 shows the simulation data and the fitted curves compared with results obtained by other researchers.

The Reynold's number based on pore diameter is

$\operatorname{Re}_{d}=\frac{\rho U d}{\mu}$

The $x, y$, and $z$ series show good agreement with each other. This indicates that the subject REV is roughly (statistically) isotropic, and contains enough primitives to be a good representation of the desired microstructure. If significant differences between the curves were observed, it would indicate that too few microstructural features were present to achieve statistically significant results, and more primitives and/or more REVs would have needed to be modeled to increase the statistical significance of the predicted property.

The present model is first compared to the unit-cube model analyzed by DeGroot and Straatman [5]. Because both approaches use idealized geometry, contrasting the two highlights only the effects of using a randomized geometry over a uniform one.

One such geometric difference is the variation in pore window size and pore window alignment. Consider, for example, the neighboring pores in Fig. 9. The left pore has 3 visible windows; two are poorly aligned, and the third is very small. The right pore, on the other hand, has 2 sizable windows, both with fair alignment with the global flow direction. The streamline coloring indicates that

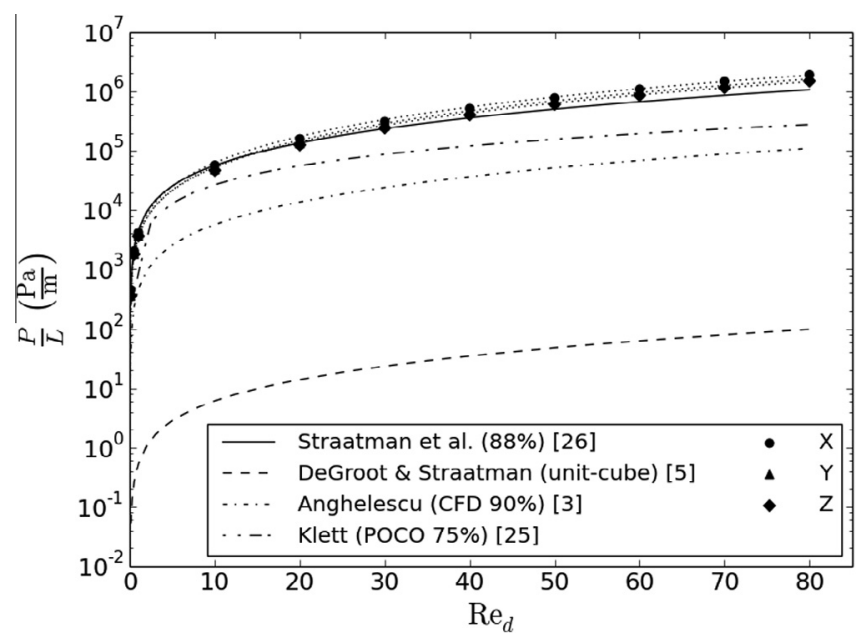

Fig. 8. Plot of pressure drop as a function of Reynolds number. Symbols indicate computed points; lines are Darcy-Forchheimer correlations reported by various authors.

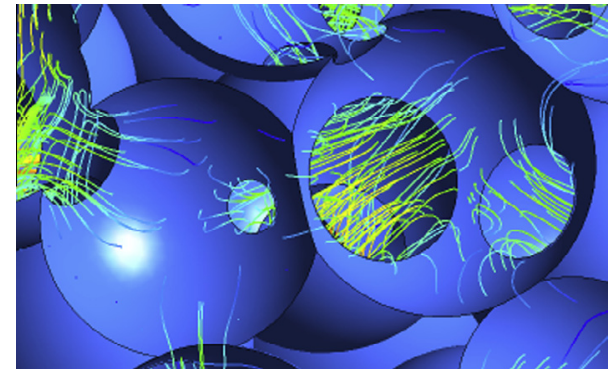

Fig. 9. Close-up of streamline plot inside the current geometry. The global flow direction is into the page. Warmer colors indicate higher velocity.

the flow much prefers the right pore to the left pore. In contrast to the velocity variations in the present analysis, each pore in the unit cube model has 3 exit windows, all misaligned from the global flow direction by the same amount. Therefore, it is not surprising that the velocity variation predicted by the unit cube model is less pronounced than that of the current model. Furthermore, it is well known that beyond Darcy flow, fluid drag, and, by extension, pressure drop varies non-linearly with velocity. Thus, the regions with higher flow speeds in the current model experience a much greater drag, resulting in a greater and more accurate overall pressure drop than that predicted using the unit-cube model.

Both the trend and the magnitudes predicted by the current model align well with results reported by other researchers. Klett et al. [25] ran experiments on $\mathrm{POCO}^{\mathrm{TM}}$ foam of $75 \%$ porosity, while the experimental results shown from Straatman et al. [26] correspond to a porosity of $88 \%$. Though the results are in reasonable correspondence with the current predictions, physical SVP porous media is known to have non-spherical pores, jagged pore windows and blocked regions, all of which will disrupt the flow. Such features will undoubtedly have an impact on the flow field, thereby influencing the observed permeability. This may serve to explain why the results for $88 \%$ reported by Straatman et al. [26] are of the same magnitude as those predicted using the present model for $80 \%$. Also included for comparison in Fig. 8 are CFD results from Anghelescu [3] based on scanned samples of $90 \%$ porosity graphite foam and DeGroot and Straatman [5] for $80 \%$ porosity foam based on the unit-cube model. While the unit cube model greatly underpredicts the pressure drop, the results of Anghelescu [3] computed on a more realistic geometry are in closer correspondence to the present modeled results. The extracted permeability and Forchheimer coefficients are given in Table 2 where they are compared with the values obtained by DeGroot and Straatman [5], and Straatman et al. [26].

\subsubsection{Energy results}

The interfacial Nusselt number in each simulation was computed using the heuristic closure proposed by Quintard [27]:

$\mathrm{Nu}_{f s}=\frac{A_{f s} h_{f s} d^{2}}{k_{f}}=\frac{\int_{A_{f s}} \mathbf{n}_{f} \cdot k_{f} \nabla T_{f} d A}{\left(\left\langle T_{s}\right\rangle^{s}-\left\langle T_{f}\right\rangle^{f}\right)} \frac{d^{2}}{k_{f}}$

Table 2

Permeability and Forchheimer coefficients.

\begin{tabular}{lll}
\hline Model & Permeability $\left(\mathrm{m}^{2}\right)$ & $\begin{array}{l}\text { Forchheimer coefficient } \\
c_{f}\end{array}$ \\
\hline $218-3^{\mathrm{a}}[26]$ & $4.46 \times 10^{-10}$ & 0.4548 \\
Unit-cube $^{\mathrm{b}}[5]$ & $3.94 \times 10^{-06}$ & 0.00347 \\
Current model $^{\mathrm{c}}$ & $5.47 \times 10^{-10}$ & 1.05
\end{tabular}

a Porosity of $88 \%$

b Values were re-fitted to Eq. (9).

c Values were averaged between three flow directions. 


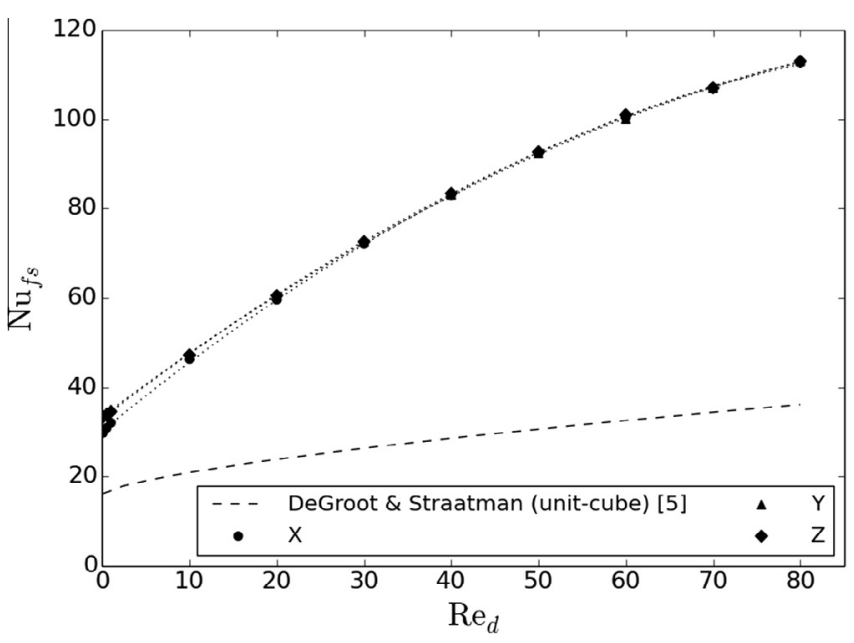

Fig. 10. Nusselt number vs. Reynold's number. Markers are computed points; lines are curve fits to Eq. (12).

where the integral term is the heat transfer into the domain, $A_{f s}$ is the surface-area-to-volume ratio of the REV, $k_{f}$ is the thermal conductivity of the fluid, and $\left\langle T_{s}\right\rangle^{s}$ and $\left\langle T_{f}\right\rangle^{f}$ are the intrinsic volume averaged temperatures of the solid and fluid phases, respectively. A surface-area-to-volume ratio of $8665 \mathrm{~m}^{-1}$ was determined using Solidworks ${ }^{\mathrm{TM}}$. The data was then fit using the numpy.polyfit function to a second order polynomial:

$\mathrm{Nu}_{f s}=a_{2} \operatorname{Re}_{d}^{2}+a_{1} \operatorname{Re}_{d}+a_{0}$

Fig. 10 shows the present simulation data and relevant curve fits in comparison with the unit-cube results of DeGroot and Straatman [5]. The average coefficients $a_{0}=32.2, a_{1}=1.53$, and $a_{2}=-0.0066$ may be inserted into Eq. (12) to give a reasonable fit with the data. Comparisons have not been made with experimental data because the interfacial Nusselt number cannot be directly compared with the average Nusselt numbers found from design scale problems such as those in $[25,26]$. The interfacial heat transfer is much larger than that predicted by DeGroot and Straatman [5] using the unitcube geometric model. A more accurate representation of the pore-level mixing is the main reason for the Nusselt number differences. Using the unit-cube geometric model, the pore-level flow is structured and spatially periodic. The flow field predicted using the current geometric model is well-mixed resulting in much higher convective heat transfer.

\section{Summary}

In this work, a novel method for generating stochastic, spatially periodic digital samples of porous media was outlined. The method requires access to DEM, CAD, and CFD software, but does not require any special equipment beyond a capable personal computer. To demonstrate the effectiveness of this approach, 12 digital samples of SVP porous media were generated using only aggregate statistical data collected from physical samples. The resulting digital samples were used in CFD simulations to predict permeability and Forchheimer coefficients as well as the constants for the Nusselt number correlation (Eq. (11)), and the data were compared to correlations obtained by other researchers. The hydraulic behavior is in very good agreement in terms of the trend with increasing Reynolds number, and is closer in magnitude than all previous modeling attempts. The remaining deviations in pressure drop between the present digital REV and actual foam samples is thought to be due to non-spherical pores, jagged pore windows, and to blocked regions, none of which are accounted for in the digital REV. The predicted convective heat transfer coefficient is much larger than that of the unit-cube model, because mixing is promoted by the random structure. The present formulation produces an accurate mathematical representation of SVP foam containing all features necessary to study all modes of heat exchange at the pore level. Combined with the fact that the current approach also incorporates variable pore size and random pore positioning, the current approach is considered most viable for studying problems involving incident radiation.

\section{Conflict of interest}

None declared.

\section{Acknowledgments}

The authors gratefully acknowledge the financial support from the Natural Sciences and Engineering Research Council of Canada (NSERC). The authors would also like to thank Dr. Jan Stransky, who coded the bubble contact law in YADE, and Dr. Bruno Chareyre and Dr. Anton Gladky for their advice and assistance in working with YADE. Finally, the authors would like to acknowledge the help Alex Kalopsis, who carried out the ANSYS CFX simulations.

\section{References}

[1] S. Haussener, P. Coray, W. Lipinski, P. Wyss, A. Steinfeld, Tomography-based heat and mass transfer characterization of reticulate porous ceramics for hightemperature processing, J. Heat Transfer 132 (2) (2010) 023305, http:// dx.doi.org/10.1115/1.4000226. Available: <http://dx.doi.org/10.1115/ 1.4000226>.

[2] B. Maruyama, J.E. Spowart, D.J. Hooper, H.M. Mullens, A.M. Druma, C. Druma, M.K. Alam, A new technique for obtaining three-dimensional structures in pitch-based carbon foams, Scr. Mater. 54 (9) (2006) 1709-1713, http:// dx.doi.org/10.1016/j.scriptamat.2005.12.060.

[3] M.S. Anghelescu, Thermal and mechanical analysis of carbon foam (ProQuest Dissertations and Theses), 2009. Available: <http://search.proquest.com/ docview/304963894?accountid=15115>.

[4] Q. Yu, B.E. Thompson, A.G. Straatman, A unit cube-based model for heat transfer and fluid flow in porous carbon foam, J. Heat Transfer 128 (4) (2006) 352-360.

[5] C.T. DeGroot, A.G. Straatman, Numerical results for the effective flow and thermal properties of idealized graphite foam, J. Heat Transfer 134 (4) (2012), http://dx.doi.org/10.1115/1.4005207. 042603-042603.

[6] P. Kumar, J. Hugo, F. Topin, J. Vicente, Influence of pore and strut shape on open cell metal foam bulk properties, AIP Conf. Proc. 1453 (1) (2012) 243-248, http://dx.doi.org/10.1063/1.4711183. Available: <http://search.ebscohost. $\mathrm{com} /$ login. aspx? direct=true $\& \mathrm{db}=\mathrm{a} 9 \mathrm{~h} \& \mathrm{AN}=75230839 \&$ site $=$ ehost-live $>$.

[7] K.C. Leong, H.Y. Li, Theoretical study of the effective thermal conductivity of graphite foam based on a unit cell model, Int. J. Heat Mass Transfer 54 (25-26) (2011) 5491-5496, http://dx.doi.org/10.1016/j.ijheatmasstransfer.2011.07. 042.

[8] L. James, S. Austin, C.A. Moore, D. Stephens, K.K. Walsh, G. Dale Wesson, Modeling the principle physical parameters of graphite carbon foam, Carbon 48 (9) (2010) 2418-2424, http://dx.doi.org/10.1016/j.carbon.2010.02.043. Available: <http://resolver.scholarsportal.info/resolve/00086223/v48i0009/ 2418_mtpppogcf>.

[9] M. Kırca, A. Gül, E. Ekinci, F. Yardım, A. Mugan, Computational modeling of micro-cellular carbon foams, Finite Elem. Anal. Des. 44 (1-2) (2007) 45-52, http://dx.doi.org/10.1016/j.finel.2007.08.008.

[10] M. Wang, N. Pan, Modeling and prediction of the effective thermal conductivity of random open-cell porous foams, Int. J. Heat Mass Transfer 51 (5-6) (2008) 1325-1331, http://dx.doi.org/10.1016/j.ijheatmasstransfer. 2007.11.031.

[11] C.C. Chueh, A. Bertei, J.G. Pharoah, C. Nicolella, Effective conductivity in random porous media with convex and non-convex porosity, Int. J. Heat Mass Transfer $71 \quad(0) \quad$ (2014) 183-188, http://dx.doi.org/10.1016/ j.ijheatmasstransfer.2013.12.041. Available: <http://www.sciencedirect.com/ science/article/pii/S0017931013010855>.

[12] W.M. Visscher, M. Bolsterli, Random packing of equal and unequal spheres in two and three dimensions, Nature 239 (5374) (1972) 504-507, http:// dx.doi.org/10.1038/239504a0.

[13] M. Tancrez, J. Taine, Direct identification of absorption and scattering coefficients and phase function of a porous medium by a Monte Carlo technique, Int. J. Heat Mass Transfer 47 (2) (2004) 373-383, http://dx.doi.org 10.1016/S0017-9310(03)00146-7. Available: http://resolver.scholarsportal. info/resolve/00179310/v47i0002/373_dioaasmbamct. 
[14] J.W. Klett, A.D. McMillan, N.C. Gallego, C.A. Walls, The role of structure on the thermal properties of graphitic foams, J. Mater. Sci. 39 (11) (2004) 3659-3676 http://dx.doi.org/10.1023/B:JMSC.0000030719.80262.f8. Available: <http:// resolver.scholarsportal.info/resolve/00222461/v39i0011/3659_trosottpogf>.

[15] D.Y. Chan, E. Klaseboer, R. Manica, Film drainage and coalescence between deformable drops and bubbles, Soft Matter 7 (6) (2011) 2235-2264.

[16] D.Y.C. Chan, E. Klaseboer, R. Manica, Theory of non-equilibrium force measurements involving deformable drops and bubbles, Adv. Colloid Interface Sci. 165 (2) (2011) 70-90, http://dx.doi.org/10.1016/j.cis.2010 12.001.

[17] ANSYS, "ANSYS v13.0; CFX v5".

[18] V. Smilauer, B. Chareyre, Yade dem formulation, V. Smilauer (Ed.) Yade Documentation (first ed.), 2010.

[19] D.P. Anderson, P.G. Wapner, D.B. Curliss, Physical property characteristics of pitch materials, Presented at MRS Proceedings, 1992.

[20] Dassault Systemes, Solidworks Educational Version 2012-2013.

[21] J.W. Klett, Process for Making Carbon Foam, 2000.
[22] ANSYS, Workbench Meshing Tool, vol. 15.0

[23] S. Whitaker, The Forchheimer equation: a theoretical development, Transp. Porous Media 25 (1) (1996) 27-61, http://dx.doi.org/10.1007/BF00141261. Available: <http://resolver.scholarsportal.info/resolve/01693913/v25i0001/ 27 tfeatd>.

[24] S.V.D. Walt, S.C. Colbert, G. Varoquaux, The NumPy array: a structure for efficient numerical computation, Comput. Sci. Eng. 13 (2)(2011) 22-30, http:// dx.doi.org/10.1109/MCSE.2011.37.

[25] J. Klett, A. McMillan, D. Stinton, Modeling geometric effects on heat transfer with graphite foam, in: Presented at 26th Annual Conference on Ceramic, Metal, and Carbon Composites, Materials, and Structures, Cocoa Beach, Florida, 2002.

[26] A.G. Straatman, N.C. Gallego, Q. Yu, L. Betchen, B.E. Thompson, Forced convection heat transfer and hydraulic losses in graphitic foam, J. Heat Transfer 129 (9) (2006) 1237-1245, http://dx.doi.org/10.1115/1.2739621.

[27] M. Quintard, Modelling local non-equilibrium heat transfer in porous media, Heat Transfer 1 (1998) 279-286. 